\title{
Josephson effects in dilute Bose-Einstein condensates
}

\author{
S. Giovanazzit, A. Smerzi and S. Fantoni \\ Istituto Nazionale di Fisica della Materia and International School for Advanced Studies, \\ via Beirut 2/4, I-34014, Trieste, Italy,
}

(October 25, 2018)

We propose an experiment that would demonstrate the "dc" and "ac" Josephson effects in two weakly linked BoseEinstein condensates. We consider a time-dependent barrier, moving adiabatically across the trapping potential. The phase dynamics are governed by a "driven-pendulum" equation, as in current-driven superconducting Josephson junctions. At a critical velocity of the barrier (proportional to the critical tunneling current), there is a sharp transition between the "dc" and "ac" regimes. The signature is a sudden jump of a large fraction of the relative condensate population. Analytical predictions are compared with a full numerical solution of the time dependent Gross-Pitaevskii equation, in an experimentally realistic situation.

PACS: 03.75.Fi,74.50.+r,05.30.Jp,32.80.Pj

The Josephson effects (JE's) are a paradigm of the phase coherence manifestation in a macroscopic quantum system [1] 3]. Observed early on in superconductors [2], JE's have been demonstrated in two weakly linked superfluid ${ }^{3} \mathrm{He}-\mathrm{B}$ reservoirs [4]. Weakly interacting Bose-Einstein condensate (BEC) gases [5] provide a further (and different) context for JE's. Indeed, magnetic and optical traps can be tailored and biased (by timedependent external probes) with high accuracy [6 8], allowing the investigation of dynamical regimes that might not be accessible with other superconducting/superfluid systems. The macroscopic BEC's coherence has been demonstrated by interference experiments [6,7], and the first evidence of coherent tunneling in an atomic array, related to the "ac" JE, has been recently reported [8].

A superconducting Josephson junction (SJJ) is usually biased by an external circuit that typically includes a current drive $I_{\text {ext }}$. The striking signatures of the Josephson effects in SJJ are contained in the voltagecurrent characteristic $\left(V-I_{e x t}\right)$, where usually one can distinguish between the superconductive branch or "dc"branch $\left(V=0, I_{e x t} \neq 0\right)$, and the resistive branch or "ac"-branch $\left(V \approx R I_{e x t}\right)$ (see for example [2]). External circuits and current sources are absent in two weakly linked Bose condensates and the Josephson effects have been related, so far, with coherent density oscillations between condensates in two traps or between condensates in two different hyperfine levels 9 14. This collective dynamical behavior is described by a non-rigid pendulum equation [9], predicting a new class of phenomena not observable with SJJ's.

Now the following question arises: can two weakly linked condensates exhibit the analog of the voltagecurrent characteristic in SJJ? Although BECs are obviously neutral, the answer is positive. A dc current-biased SJJ can be simulated by considering a tunneling barrier moving with constant velocity across the trap. At a critical velocity of the barrier a sharp transition between the "dc" and "ac" (boson) Josephson regimes occurs. This transition is associated with a macroscopic jump in the population difference, that can be easily monitored experimentally by destructive or non-destructive techniques.

In the following we will briefly introduce the phenomenological equations of the resistively shunted junction (RSJ) model for the SJJ. We will describe the corresponding experiment for two weakly linked BECs and show that the relevant equations are formally equivalent to the RSJ equations. Then we compare the analytical results with a numerical integration of the Gross-Pitaevskii equation in a realistic 3D setup.

In the RSJ model, SJJ is described by an equivalent circuit [2] in which the current balance equation is

$$
I_{c} \sin (\theta)+G V+C \dot{V}=I_{e x t}
$$

where $I_{c}$ is the upper bound of the Josephson supercurrent $I$ (which is represented, in the ideal case, by the sinusoidal current-phase relation $I=I_{c} \sin (\theta)$ ); $G$ is an effective conductance (offered by the quasiparticles and the circuit shunt resistor), and $C$ is the junction capacitance. The voltage difference $V$ across the junction is related to the relative phase $\theta$ by

$$
\dot{\theta}=2 e V / \hbar \text {. }
$$

In the low conductance limit $G \ll \omega_{p} C$ where $\omega_{p}=$ $\sqrt{2 e I_{c} / \hbar C}$ is the Josephson plasma frequency, combining equations (11) and (2) leads to the "driven pendulum" equation

$$
\ddot{\theta}=-\omega_{p}^{2} \frac{\partial}{\partial \theta} U(\theta)
$$

where $U$ is the tilted "washboard" potential:

$$
U(\theta)=1-\cos (\theta)+i \theta
$$

with $i=I_{e x t} / I_{c}$. This equation describes the transient behavior before the stationary dissipative behavior is reached (resistive branch). If we start from equilibrium, with $i=0$, and increase adiabatically the current, 
no voltage drop develops until the critical value $i=1$ is reached (neglecting secondary quantum effects). At this point $V$ continuously develops until a stationary asymptotic dissipative behavior is reached in a time scale approximately of order $C / G$. Similar phenomenology may occur in BECs and we will derive equations formally identical to Equations (3) and (位).

A weak link between two condensates can be created by focusing a blue-detuned far-off-resonant laser sheet into the center of the magnetic trap [6]. The weak link can be tailored by tuning the width and/or the height of the laser sheet. Raman transitions between two condensates in different hyperfine levels provide a different weak link [7], in analogy with the "internal Josephson effect" observed in 70 s with ${ }^{3} \mathrm{He}-\mathrm{A}[\mathrm{15}$.

Here we consider a double well potential in which the laser sheet slowly moves across the magnetic trap with velocity $v$ (but our framework can be easily adapted to investigate the internal Josephson effect). In the limit of very low $v$, the two condensates remain in equilibrium, i.e. in their instantaneous ground state, because of the non-zero tunneling current that can be supported by the barrier. In fact, an average net current, proportional to the velocity of the laser sheet, flows through the barrier, sustained by a constant relative phase between the two condensates. This keeps the chemical potential difference between the two subsystems locked to zero, as in the SJJ dc-branch. However, the superfluid component of the current flowing through the barrier is bounded by a critical value $I_{c}$. As a consequence there exists a critical velocity $v_{c}$, above which a non-zero chemical potential difference develops across the junction. This regime is characterized by a running-phase mode, and provides the analog of the ac-branch in SJJ's.

The "dc" and "ac" BEC regimes are governed by a phase-equation similar to the current-driven pendulum equations (3) and (4). Such equations together with the sinusoidal current-phase relation $I=I_{c} \sin (\theta)$ describe the phase difference and current dynamics. The dimensionless current $i$ is related to the barrier velocity by

$$
i=v / v_{c}
$$

with the critical velocity $v_{c}$ given by

$$
v_{c}=\frac{\hbar \omega_{p}^{2}}{F}
$$

where $F$ is to a good approximation represented by double the average force exerted by the magnetic trap on single atoms in one well.

Equations (3)-(6) can be derived by a time-dependent variational approximation and have also been verified, as we discuss below, by the full numerical integration [18] of the Gross-Pitaevskii equation (GPE) [16, 17]. The GPE describes the collective dynamics of a dilute Bose gas at zero temperature:

$$
i \hbar \frac{\partial}{\partial t} \Psi=\left[H_{0}(t)+g|\Psi|^{2}\right] \Psi
$$

where $H_{0}(t)=-\frac{\hbar^{2}}{2 m} \nabla^{2}+V_{\text {ext }}(\mathbf{r}, t)$ is the non interacting Hamiltonian and where $g=4 \pi \hbar^{2} a / m$, with $a$ the scattering length and $m$ the atomic mass. The order parameter $\Psi=\Psi(\mathbf{r}, t)$ is normalized as $\int d \mathbf{r}|\Psi(\mathbf{r}, t)|^{2}=N$, with $N$ the total number of atoms. The external potential is given by the magnetic trap and the laser barrier $V_{\text {ext }}(\mathbf{r}, t)=V_{\text {trap }}(\mathbf{r})+V_{\text {laser }}(z, t)$. We consider a harmonic, cylindrically symmetric trap $V_{\text {trap }}(\mathbf{r})=$ $\frac{1}{2} m \omega_{r}^{2}\left(x^{2}+y^{2}\right)+\frac{1}{2} m \omega_{0}^{2} z^{2}$ where $\omega_{r}$ and $\omega_{0}$ are the radial and longitudinal frequency, respectively. The barrier is provided by a Gaussian shaped laser sheet, focused near the center of the trap $V_{\text {laser }}(z)=V_{0} \exp \left(-\left(z-l_{z}\right)^{2} / \lambda^{2}\right)$ with the coordinate $l_{z}(t)$ describing the laser motion and $v=d l_{z} / d t$ its velocity.

The equations (3) to (6) can be derived by solving variationally the GPE using the ansatz: $\Psi(\mathbf{r}, t)=$ $c_{1}(t) \psi_{1}(\mathbf{r})+c_{2}(t) \psi_{2}(\mathbf{r})$, where $c_{n}=\sqrt{N_{n}(t)} \exp \left(i \theta_{n}(t)\right)$ are complex time-dependent amplitudes of the left $n=1$ and right $n=2$ condensates (see also [9]). The trial wave functions $\psi_{1,2}(\mathbf{r})$ are orthonormal and can be interpreted as approximate ground state solutions of the GPE of the left and right wells. The equations of motion for the relative population $\eta=\left(N_{2}-N_{1}\right) / N$ and phase $\theta=\theta_{2}-\theta_{1}$ between the two symmetric traps are

$$
\begin{aligned}
& \hbar \dot{\eta}=\left(2 E_{J} / N\right) \sqrt{1-\eta^{2}} \sin (\theta), \\
& \hbar \dot{\theta}=F l_{z}(t)-\frac{2 E_{J}}{N} \frac{\eta}{\sqrt{1-\eta^{2}}} \cos (\theta)-\frac{N E_{c}}{2} \eta
\end{aligned}
$$

where $E_{c}=2 g \int d \mathbf{r} \psi_{1}(\mathbf{r})^{4}$ is the variational ana$\log$ of the capacitive energy in SJJ, while $E_{J}=$ $-N \int d \mathbf{r} \psi_{1}(\mathbf{r})\left[H_{0}+g N \psi_{1}^{2}(\mathbf{r})\right] \psi_{2}(\mathbf{r})$ is the Josephson coupling energy. The current-phase relation $I=$ $I_{c} \sqrt{1-\eta^{2}} \sin (\theta)$ is directly related to Eq. (8) where the critical current is given by $I_{c}=E_{J} / \hbar$. $F l_{z}(t)$ represents the contribution to the chemical potential difference in the two wells due to the laser displacement $l_{z}$ (after linearizing in $l_{z}$ ), and where $F=\int d \mathbf{r}\left(\psi_{1}(\mathbf{r})^{2}-\psi_{2}(\mathbf{r})^{2}\right) \frac{\partial}{\partial l_{z}} V_{\text {laser }} \simeq$ $m \omega_{0}^{2} \int d \mathbf{r} z\left(\psi_{1}(\mathbf{r})^{2}-\psi_{2}(\mathbf{r})^{2}\right)$. The above variational method provides a simple and useful interpolating scheme between the low interacting limit $N^{2} E_{c} \ll E_{J}$ and the opposite limit $N^{2} E_{c} \gg E_{J}$. In the last case, and with $\eta \ll 1$, we recover the driven-pendulum phase equation (3) and the critical velocity relations (5) and (6) with $\hbar \omega_{p}=\sqrt{E_{J} E_{c}}$. In particular, it is legitimate to consider the Josephson coupling as a perturbation, with the the phase dynamics entirely determined by the difference in the chemical potentials $\mu_{1}\left(N_{1}, l_{z}\right)$ and $\mu_{2}\left(N_{2}, l_{z}\right)$ in the two wells. In this case $E_{c}$ corresponds to $2\left(\partial \mu_{1} / \partial N_{1}\right)_{l_{z}}$ and $\hbar^{2} \omega_{p}^{2}=E_{J}\left(\partial \mu_{1} / \partial N_{1}\right)_{l_{z}}$. The critical velocity is proportional to the critical current: $v_{c}=\left(\frac{d N_{1}}{d l_{z}}\right)^{-1} I_{c}$, with 


$$
\left(\frac{d N_{1}}{d l_{z}}\right)^{-1}=\left(\frac{\partial \mu_{1}}{\partial l_{z}}\right)_{N_{1}}^{-1}\left(\frac{\partial \mu_{1}}{\partial N_{1}}\right)_{l_{z}}
$$

and $\left(\partial \mu_{1} / \partial l_{z}\right)_{N_{1}}$ being $F / 2$ in Eq.(6). These derivatives can be computed numerically. In the Thomas-Fermi (TF) limit they reduce to

$$
\left(\frac{\partial \mu_{1}}{\partial N_{1}}\right)_{l_{z}}=\frac{g}{V_{T F}}
$$

and

$$
\left(\frac{\partial \mu_{1}}{\partial l_{z}}\right)_{N_{1}}=\frac{1}{V_{T F}} \int_{V_{T F}} d \mathbf{r} \frac{\partial}{\partial l_{z}} V_{\text {laser }}
$$

where $V_{T F}$ is the volume of the region in which $\Psi_{1}$ is different from zero (in the $\mathrm{TF}$ approximation).

We make the comparison of Eqs. (8) and (9) with a full numerical integration of the GPE in an experimentally realistic geometry relative to the limit $N^{2} E_{c} \gg E_{J}$. In particular, we show that Eq. (6), derived in the limit of $\eta \ll 1$, still remains a good approximation even for $\eta \approx 0.4$. The details of the numerical calculation are given elsewhere [18].

We have considered the JILA setup, with $N=5 \times 10^{4}$ $\mathrm{Rb}$ atoms in a cylindrically symmetric harmonic trap, having the longitudinal frequency $\omega_{0}=50 \mathrm{~s}^{-1}$ and the radial frequency $\omega_{r}=17.68 \mathrm{~s}^{-1}$. The value of the scattering length considered is $a=58.19 \dot{A}$. A Gaussian shaped laser sheet is focused in the center of the trap, cutting it into two parts. We assume that the (longitudinal) $1 / e^{2}$ half-width of the laser barrier is $3.5 \mu \mathrm{m}$ and the barrier height $V_{0} / \hbar=650 \mathrm{~s}^{-1}$.

Although the lifetime of a trapped condensate can be as long as minutes, we have made a quite conservative choice, by considering a time scale on the order of one second. The possibility to perform experiments on a longer time-scale will improve the observability of the phenomena we are discussing. With this choice of time scale, that corresponds only to few plasma oscillations, an adiabatic increase of the velocity is not possible, therefore we proceed as follows. For $t<0$ the laser is at rest in the middle of the trap, $l_{z}=0$, and the two condensates are in equilibrium. For $t>0$ the laser moves across the trap, with constant velocity, and the relative atomic population is observed at $t_{f}=1 \mathrm{~s}$. With this initial condition, which introduces small plasma oscillations in the relative population, it is expected, in absence of dissipation, to slightly reduce the critical current by the numerical factor $\approx 0.725$ (see the general properties of the driven pendulum equation [2]).

In Fig. 1 we show the relative condensate population $\eta=\left(N_{2}-N_{1}\right) / N$, calculated after 1 second, for different values of the laser velocity $v$. The crosses are the results obtained with the full numerical integration of the time-dependent GPE (7). The dot-dashed line shows the equilibrium values $\eta_{e q}$ of the relative population calculated with the stationary GPE and with the laser at rest in the "final" position $l_{z}=v t_{f}$. The displacement of $\eta\left(t_{f}\right)$ from $\eta_{e q}$ is a measure of the chemical potential difference, being $\Delta \mu=\mu_{2}-\mu_{1} \approx N E_{c}\left(\eta\left(t_{f}\right)-\eta_{e q}\right) / 2$.

For $v<0.42 \mu \mathrm{m} / \mathrm{s}$, the atoms tunnel through the barrier in order to keep the chemical potential difference $\Delta \mu$ locked around zero. The dc component of the tunneling current is accounted for by an averaged constant phase difference between the two condensates. This is the close analog of the dc Josephson effect in superconducting Josephson junctions. The small deviations between the dashed line and the crosses are due to the presence of plasma oscillations (induced by our initial condition). At $v \approx 0.42 \mu \mathrm{m} / \mathrm{s}$ there is a sharp transition, connected with the crossover from the dc-branch to the ac-branch in SJJ. For $v>0.42 \mu \mathrm{m} / \mathrm{s}$, the phase difference starts running and the population difference, after a transient time, remains on average fixed. A macroscopic chemical potential difference is established across the junction. In this regime ac oscillations in the population difference are observed. The frequency of such oscillations are approximatively given by $\Delta \mu(t) / \hbar$ (not visible in the figure).



FIG. 1. Fractional population imbalance versus the velocity of the laser creating the weak link. A sharp transition between the "dc" and the "ac" branches occurs at a barrier critical velocity. The solid line and the crosses are the analytical and the numerical calculations, respectively. The dashed-dot line represents the static equilibrium value $\eta_{e q}$ calculated with the center of the laser at $v t_{f}$.

The solid line of Fig.1 corresponds to the solutions of Eqs. (8) and (9) in which the value of the energy integrals $E_{c} N / \hbar=2.46 \mathrm{~ms}^{-1}$ and $E_{J} / N \hbar=2.41 \times 10^{-4} \mathrm{~ms}^{-1}$ are chosen in order to give the correct value of $\omega_{p}=$ $2.44 \times 10^{-2} \mathrm{~ms}^{-1}$ and $I_{c}=12.1 \mathrm{~ms}^{-1}$. The values $\omega_{p}, I_{c}$ are calculated numerically studying the frequency of small oscillations around equilibrium and the current-phase relation, respectively. The force integral is 
$F / \hbar=1.060 \mathrm{~ms}^{-1} \mu \mathrm{m}^{-1}$. The parameters $\omega_{p}, I_{c}$ and $F$ are calculated with the laser at rest $(v=0)$ in $l_{z}=0$. Using these values in Eq. (6) and taking into account the reducing factor 0.725 we obtain the value $0.407 \mu \mathrm{m} \mathrm{s} \mathrm{s}^{-1}$ for the critical velocity, in agreement with the value observed in the simulation.

Small deviations between the variational solutions (full line in Fig.1) and the numerical results (crosses in Fig.1), above the critical velocity, are due to "level-crossing" effects. Numerical results [18] show that when the condensate ground state of the "upper" well is aligned with the excited collective dipole state in the "lower" well, a finite number of atoms go from the "upper" well to the "lower" well. Close to this tunneling resonance it is possible to control, by manipulating the barrier velocity below a fraction of $v_{c}$, the dc flux of atoms from the ground state condensate in the "upper" well to the longitudinal intrawell collective dipole mode of the condensate in the "lower" well. This effect is directly observable in the macroscopic longitudinal oscillations of the two condensates (at frequencies $\approx \omega_{0}$ ).

Concerning a possible realization of the phenomenon described in this work, we note that for small barrier velocities $v$, the motion of the laser sheet with respect to the magnetic trap with velocity $v$ or, viceversa, the motion of the magnetic trap with velocity $-v$, are equivalent, there being negligible corrections due to different initial accelerations.

Thus far we have discussed the zero temperature limit. At finite temperature dissipation can arise due to incoherent exchange of thermal atoms between the two wells. This can be described phenomenologically by including a term $-E_{c} G \dot{\theta} / \omega_{p}^{2}$ in Eq. (3) where $G$ is the conductance. Dissipation will be negligible as long as the characteristic time scale $\left(E_{c} G\right)^{-1} \approx(20 G / \hbar) s$ is bigger than the time scale of the experiment $(\approx 1 s)$.

To conclude we note that while it could be difficult to measure directly the plasma oscillations, since their amplitude is limited by $\Delta \eta<\frac{4}{N} \sqrt{\frac{E_{J}}{E_{c}}}$, the macroscopic change in the population difference may be easily detected with standard techniques. Moreover the framework that we have discussed can be easily adapted to investigate the internal Josephson effect.

Our phenomenological equations are similar to the driven pendulum equation governing the Josephson effects in SJJs. As a consequence, within this framework we can study the "secondary quantum phenomena", such as the Macroscopic Quantum Tunneling between different local minima of the washboard potential (see for instance 19]).

It is a pleasure to thank L. P. Pitaevskii, S. Raghavan and S. R. Shenoy for many fruitful discussions.
* Present Address: Department of Chemical Physics, Weizmann Institute of Science, 76100 Rehovot, Israel.

[1] P. W. Anderson, Basic Notions of Condensed Matter Physics (Benjamin-Cummings, Menlo Park, 1984).

[2] A. Barone and G. Paterno, Physics and Applications of the Josephson Effect (Wiley, New York, 1982).

[3] A. Barone, NATO ASI Series Quantum Mesoscopic Phenomena and Mesoscopic Devices in Microelectronics, Ankara June 1999, (I.O. Kulik and R. Ellialtioglu Eds.) Kluwer (in press).

[4] O. Avenel, and E. Varoquaux, Phys. Rev. Lett. 55, 2704 (1985); S. V. Pereverzev et al., Nature 388, 449 (1997); S. Backhaus, et al., Science 278, 1435 (1998); S. Backhaus, et al., Nature 392, 687 (1998).

[5] M. H. Anderson et al., Science 269, 198 (1995); K. B. Davis, et al., Phys. Rev. Lett. 75, 3969 (1995); C. C. Bradley, et al., Phys. Rev. Lett. 75, 1687 (1995); D. G. Fried, et al., Phys. Rev. Lett. 81, 3811 (1998).

[6] M. R. Andrews et al., Science 275, 637 (1997).

[7] D. S. Hall et al., 81, 1539, 1543 (1998).

[8] B. P. Anderson and M. A. Kasevich, Science 282, 1686 (1998).

[9] A. Smerzi, S. Fantoni, S. Giovanazzi, and S. R. Shenoy, Phys. Rev. Lett., 79, 4950 (1997); S. Raghavan, A. Smerzi, S. Fantoni, and S. R. Shenoy, Phys. Rev. A, 59, 620 (1999).

[10] C. J. Milburn, J. Corney, E. M. Wright, and D. F. Walls, Phys. Rev. A 55, 4318 (1997).

[11] J. Ruostekoski and D.J. Walls, Phys. Rev. A 58 R50 (1998)

[12] P. Villain and M. Lewenstein, Phys. Rev. A 59, 2250 (1999).

[13] I. Zapata, F. Sols, and A. Leggett, Phys. Rev. A 57, R28 (1998).

[14] J. Williams, R. Walser, J. Cooper, E. Cornell, and M. Holland, Phys. Rev. A 59, R31 (1999).

[15] R. A. Webb et al., Phys. Lett 48A, 421 (1974); Phys. Rev. Lett 33, 145 (1974); A. J. Leggett, Rev. Mod. Phys. 47, 331 (1975); K. Maki and T. Tsuneto, Prog. Theor. Phys. 52, 773 (1974).

[16] L. P. Pitaevskii, Sov. Phys. JETP, 13, 451 (1961); E. P. Gross, Nuovo Cimento 20, 454 (1961); J. Math. Phys. 4, 195 (1963).

[17] F. Dalfovo, S. Giorgini, L. P. Pitaevskii and S. Stringari, Rev. Mod. Phys. 71, 463 (1999).

[18] S. Giovanazzi, Ph.D. Thesis, SISSA, Trieste, Italy, (1998), unpublished.

[19] G. Schon, and A. D. Zaikin, Phys. Rep. 198, 237 (1999); P. Silvestrini, B. Ruggiero and A. Esposito, Low Temp. Phys. 22, 195 (1996). 